\title{
PROFESORADO PRINCIPIANTE: ENTRE MONTAÑAS Y VEREDAS, APRENDIENDO A SER MAESTRO EN EL SURESTE MEXICANO
}

\section{BEGINNING TEACHERS: BETWEEN MOUNTAINS AND SIDEWALKS, LEARNING TO BE A TEACHER IN SOUTHEAST MEXICO}

Iván Alexis Pinto Diaz*

\begin{abstract}
Resumen: Resultado del trabajo de campo de la investigación "los primeros años de ejercicio docente en educación básica. Reconstrucción de prácticas y experiencias en contextos desfavorecidos”, se presenta evidencias de la situación que enfrentan los profesores principiantes en las comunidades rurales del estado de Chiapas, en el sureste mexicano. Se recupera las peripecias, angustias y los aprendizajes de los primeros días y meses que como profesores principiantes viven en el centro de trabajo y la comunidad. A partir de una serie de entrevistas in situ se documenta la experiencia, pero sobre todo se desentraña la distancia entre los procesos de formación que ofrecen las instituciones formadoras de profesores y la realidad que les toca vivir a los nuevos trabajadores de la educación; se destaca que no existe apoyo y acompañamiento para esos primeros momentos del ejercicio docente. Se hace evidente y como lo señalan los profesores, es la realidad lo que los forma y los compromete con la actividad profesional en la que se encuentran inmersos. El trabajo forma parte de una intención mayor, la de documentar la realidad y participar en el debate sobre la necesidad de establecer programas y apoyos para que los profesores principiantes se desarrollen y consoliden en la tarea docente. Como se notará, los profesores principiantes viven intensamente sus primeros contactos con su profesión (a pesar de las penurias y conflictos), es una experiencia reveladora y significativamente formativa, la cuestión es sistematizarla y presentarla como un recurso que puede potencializar la formación continua.
\end{abstract}

Palabras clave: Profesores principiantes. Dificultades. Retos. Formación.

\begin{abstract}
This article presents the rresults of the investigation called "The first years of teaching in basic education: reconstruction of practices and experiences in disadvantaged contexts" which presents evidence of the situation faced by beginning teachers in rural communities in the state of Chiapas in the southeast of Mexico.The article recovers the adventures, anxieties and lessons from the first days and months that beginning teachers experience in the workplace, and in the community. From a series of on-site interviews the article reports not only the experience, but also the gap between the training processes offered by teacher education institutions, and the reality lived by beginning teachers. The article emphasizes that there is not support, and assistance for the first years of teaching. It also provides evidence based on teachers' testimonies, that it is the reality they experience that turns them into teachers and, commits them to the profession in which they are immersed. The article has a broader purpose, which is to document the reality, and participate in the debate about the need to establish, and support programs for beginning teachers to develop and strengthen the teaching task. As it will be noticed,
\end{abstract}

\footnotetext{
* Maestro en Educación Superior. Universidad Autónoma de Chiapas. México. Maestro en Educación con especialidad en Investigación Educativa. Universidad Autónoma de Chiapas. México. Candidato a Doctor en Educación. Universidad Pedagógica Nacional. México. Profesor de la Universidad Autónoma de Chiapas. E-mail: <iapidiaz70@hotmail.com>.

* Master in Education. Autonomous University of Chiapas. Mexico. Master in Education with a degree in Educational Research. Autonomous University of Chiapas. Mexico. Doctoral student in Education. National Pedagogy University. Mexico. Professor at the Autonomous University of Chiapas. E-mail: <iapidiaz70@hotmail.com>.
} 
beginning teachers live intensively their first teaching years, despite the difficulties and conflicts, it is a revealing and formative meaningful experience. The issue is systematized and presented as a resource that can enrich continuing education.

Keywords: Beginning teachers. Difficulties. Challenges. Education.

\section{Introducción}

Este trabajo es producto de las consideraciones desarrolladas en el marco del proyecto de investigación no. 146031, denominado "Los primeros años de ejercicio docente en educación básica. Reconstrucción de prácticas y experiencias en contextos desfavorecidos" que se lleva a cabo por académicos y estudiantes de la Universidad Pedagógica Nacional -Unidad Ajusco- ${ }^{1}$ en el marco de la convocatoria SEB-2009-1 del Consejo Nacional de Ciencia y Tecnología del gobierno mexicano.

La investigación tiene como propósito "analizar cualitativamente las prácticas, dificultades y logros de los maestros noveles de educación primaria y secundaria” en contextos desfavorecidos y rurales. Para el estudio se consideró a profesores de dos entidades mexicanas, una de ellas, y es a lo que refiere este trabajo, tiene que ver con profesores principiantes del estado de Chiapas.

Poco se ha investigado en el contexto mexicano, la realidad que enfrentan los recién egresados de las instituciones formadoras de profesores, cuando viven sus primeras experiencias como trabajadores de la educación. Cómo y qué define la experiencia entre pasar de la tranquilidad de ser estudiante normalista y el proceso de hacerse maestro. El estudio observa con

\footnotetext{
${ }^{1}$ La investigación es coordinada por la Dra. Etelvina Sandoval y la Mtra. Alicia Carvajal. En dicho proyecto colaboramos alumnos de maestría y doctorado de la citada universidad. El autor de este reporte coordina el trabajo de campo en la entidad chiapaneca.
}

detenimiento la configuración del estilo docente en los primeros años de ejercicio, en tanto es un momento crucial por la complejidad de elementos que lo permean, y entre los cuales se encuentra la historia vivida por el sujeto en la educación Normal, el origen social de los profesores y el contexto donde se ubica la experiencia del ejercicio profesional.

Se retoma el planteamiento de considerar los inicios de la profesión docente como una fase de intenso aprendizaje en el oficio del magisterio, donde se va conformando imágenes de la profesión y generando prácticas específicas, la cual forma parte de un continuo aprendizaje a lo largo de la vida (SANDOVAL, 2011).

También se asume que

La inserción profesional en la enseñanza (...) es el periodo de tiempo que abarca los primeros años, en los cuales los profesores han de realizar la transición desde estudiantes a docentes. Es un periodo de tensiones $\mathrm{y}$ aprendizajes intensivas en contextos generalmente desconocidos y durante el cual los profesores principiantes deben adquirir conocimiento profesional además de conseguir mantener un cierto equilibrio personal. (MARCELO GARCÍA, 2008, p. 14).

El trabajo se orienta desde una perspectiva metodológica cualitativa con énfasis en enfoques narrativos y análisis de trayectorias profesionales (BOLIVAR, 2001; TARRÉS, 2004). 
Para el estudio se consideró a los profesores que se inician en escuelas de Chiapas, ${ }^{2}$ ya que esa entidad del sureste de México ofrece elementos de gran valía para conocer y reflexionar la configuración del proceso de hacerse maestro en contextos rurales y marginados. Esa entidad tiene marcado rezago social y económico. Una alta dispersión de comunidades y los problemas educativos son cuestiones recurrentes.

\section{La educación Normal, las condiciones de la formación para el ejercicio profesional}

En Chiapas, existen diecinueve instituciones formadoras de profesores (las cuales llevan la denominación de escuelas normales) para la educación básica (preescolar, primaria y secundaria). De éstas, solamente una ofrece alguna formación con carácter rural. ${ }^{3}$ Pero en su conjunto, dichas instituciones se encargan de formar en elementos de orden académico, es decir, en preparación para estar presente en aula y atender los contenidos del nivel educativo que se trate (se deja de lado la formación para entender e incursionar en el ámbito comunitario, es decir, no se prepara para adaptarse al contexto laboral).

\footnotetext{
${ }^{2}$ Se entrevistaron a cuatro profesores de educación primaria del municipio de La Trinitaria, siete profesores de educación secundaria, de los cuales cinco pertenecen al Centro de Educación Básica del Estado de Chiapas (CEBECH) de la ciudad rural sustentable "Juan del Grijalva” en el municipio de Ostuacán, uno de la localidad Golonchan viejo del municipio de Chilón y uno más del municipio de Oxchuc.

${ }^{3}$ En México existen dos tipos de normales, las urbanas y rurales. De las escuelas normales rurales solo quedan 16 en el país, de éstas una en Chiapas. La formación de profesores con conocimiento e identidad del contexto rural es cada vez menor, en tanto las instituciones que forman para este campo son pocas y con una reducida matrícula.
}

En el sistema educativo mexicano, los normalistas atienden las escuelas de la educación básica (en años recientes, la presencia de universitarios es más frecuente; su intervención es distante de los requerimientos necesarios para atender las necesidades de los estudiantes y las escuelas). Las normales de licenciatura en educación preescolar preparan a profesoras para el jardín de niños (de tres grados); las normales que ofrecen la licenciatura en educación primaria preparan para atender las escuelas primarias (de seis grados) y las normales superiores forman para atender el nivel de secundaria (se estudia en tres grados).

Aquí cabe un comentario como antecedente en el análisis de la situación en las normales mexicanas y específicamente para entender los procesos formativos que las caracterizan. Desde 1984 la educación Normal fue "elevada" al modelo y nivel de institución de educación superior (IES), lo cual implicó que pasaran del tradicional modelo de organización que los caracterizaba desde su origen, es decir, con procesos orientados en formar a los futuros maestros como técnicos de la enseñanza priorizando el saber hacer del ejercicio docente, para pasar a un modelo de trabajo y organización escolar en donde se impuso dos actividades sustantivas (a parte de la docencia): la investigación y la difusión.

Antes de 1984 los formadores que colaboraban en las normales, se dedicaban (como tarea central del contrato laboral) a impartir clase y orientar a los alumnos en cómo definir un estilo docente en la educación básica; posterior a ese año, los formadores los acicatearon para que entraran en una "competencia” para concursar por las plazas docentes del nuevo modelo de escuela Normal (que las vinculaban a la educación superior mexicana), plazas que imponían nuevas tareas y compromisos. 
Los formadores de docentes de las normales dejaron (porque así se los impuso la nueva política y modelo de escuela determinado oficialmente) de tener como eje de su atención el trabajo de preparación para formar técnicos para la enseñanza, empezaron a preocuparse por las demás actividades, que no conocían y que tenían que realizar para cumplir con las condiciones organizativas y laborales de la institución normalista como IES. Dentro de estas actividades, se encontraban las descargas académicas, el formador ya no se ocupaba solo de dar clases, ahora se le imponía cierto tiempo para la investigación o la difusión. La descarga académica fue un tiempo perdido, en tanto el formador no se encontraba preparado para desarrollarla, pero significó al cabo del tiempo la posibilidad de realizar cualquier otra cosa, menos actividades que fortalecieran la vida académica de las normales y los procesos de formación de los estudiantes. Empezó a perderse la esencia formativa que preparaba para la docencia.

Para los años recientes, la tendencia de las políticas neoliberales del gobierno mexicano ha sido la de constreñir el financiamiento al normalismo mexicano, hay una orientación a reducir su presencia como institución formadora, algunas voces oficialistas plantean su desaparición o dejarlas que vayan muriéndose por inanición.

Aunado a lo anterior, y en la lógica de que la escuela Normal es una IES, los formadores que prestan servicios en dichas instituciones tienden a ser más universitarios y menos normalistas. Las prácticas y orientaciones se inscriben en la lógica universitaria, en detrimento de la tradicional concepción del normalismo mexicano, es decir, preparar para la enseñanza en las comunidades rurales mexicanas.

Los anteriores elementos son nodales para entender la situación de los egresados de las normales, cuando se enfrentan al complejo mundo del trabajo y las primeras experiencias como profesores de la educación básica. Y es que la situación que se observa en la formación de los profesores, está caracterizada por la falta de preparación para entender el ámbito social y cultural de las comunidades en donde le tocará incursionar al egresar. $\mathrm{Y}$ bajo esta consideración, el primer elemento a comentar y reflexionar en este trabajo es la llegada al mundo laboral de los egresados de las normales.

\section{De la educación Normal a la inserción laboral}

La obtención de la plaza se inscribe en la lógica de presentar examen de oposición que, en los años recientes, se ha convertido en una condición para tener trabajo en el magisterio mexicano. Al examen asisten tanto egresados de las normales como de universidades, y los rezagados en la obtención de una plaza de docente. En la convocatoria y la presentación del examen, el número de aspirantes rebasa por mucho la oferta laboral. La expectativa y el primer momento de conflicto del recién egresado, profesor graduado y aspirante a trabajador contratado, es el de competir con una cantidad exorbitante de compañeros por el trabajo (una plaza de profesor que otorga el gobierno). La incertidumbre, después de cuatro años de ser alumno de una Normal, concluye en el momento de publicación de los resultados de quienes se irán a trabajar en cualquiera de las escuelas de educación básica de Chiapas.

Los cuatro años de formación de la Normal trascurren en la tranquilidad de las aulas y las prácticas para aprender del hacer del profesor, prácticas que se desarrollan en lugares circunvecinos de la institución 
que los forma (el plan de estudios establece que el último año de la licenciatura se desarrolle combinando trabajo docente por periodos prolongados en escuelas primarias y periodos cortos de estudio en la Normal); la experiencia estudiantil se circunscribe a estudiar y acreditar las materias. No hay duda que de una u otra manera existe exigencia en la formación, pero no se ofrece elementos para que el futuro profesor comprenda y se arraigue en la comunidad. Poco pasa por el pensamiento del estudiantado el lugar en donde les tocará iniciarse como profesores, en tanto que la institución que los forma soslaya una formación que los prepare en lo elemental de cómo asumir la tarea docente en un contexto que no sea el urbano, por lo que la inserción a la escuela y las comunidades de las apartadas zonas rurales se convierte en un asunto personal y que se definirá hasta que llegue el momento en que el profesor se enfrente a dicha realidad.

\section{El viaje a la comunidad: los primeros problemas}

Figura 1 - Jolja, Tila, México. Una comunidad para profesor principiante.

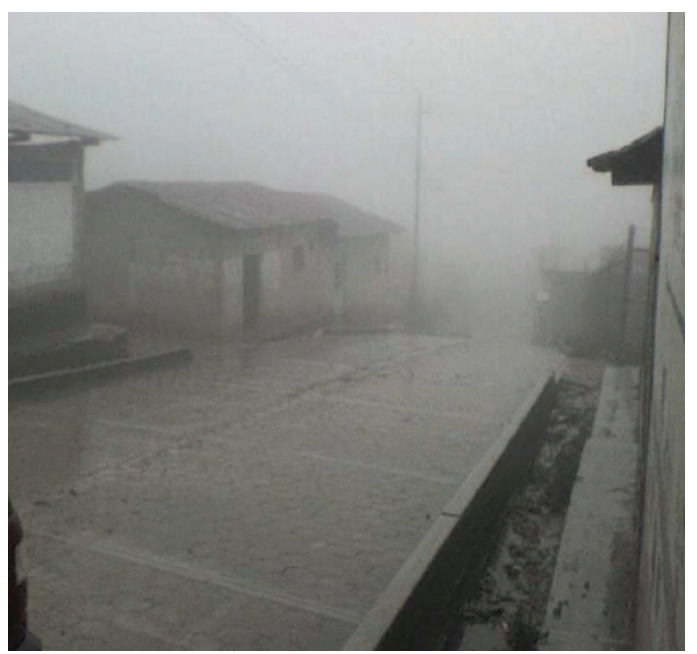

Pasada la incertidumbre y teniendo la plaza asignada, todo egresado de normales es enviado a una comunidad rural, dependiendo el nivel y la modalidad, el lugar de asignación es definido por costumbres y prácticas construidas por los profesores que ya tienen experiencia y años de servicio, entre éstas se encuentran dar prioridad a las mujeres o por el número que le corresponde en la lista de quien llega primero a la supervisión escolar, hasta por el estado de ánimo de quien en ese momento le corresponde asignar los lugares; ${ }^{4}$ estas son de las primeras condiciones con las que tienen que lidiar los nuevos profesores, pero también se constituyen en elementos que preparan para que en el futuro sepan que tienen derechos y que hay un sindicato que los defiende, en virtud de que las condiciones de asignación de escuelas los dejan inconformes e inician una búsqueda de razones y argumentos para que no les vuelva a pasar y se les respete como trabajadores. Pero también marca el camino de la competencia y la de cultivar relaciones para mejorar el lugar de asignación.

El anhelo, pero sobre todo la necesidad ${ }^{5}$ de tener trabajo seguro, hace que los noveles profesores (y por la velocidad en que corre el tiempo entre que pasa el examen, saben el resultado y tienen que empezar el complejo proceso administrativo para la afiliación) no comprendan la magnitud de lo que les

\footnotetext{
${ }^{4}$ Una de las profesoras entrevistadas refiere que cuando se le otorgó la orden de comisión y se presentó ante el supervisor, éste le indicó que por esa ocasión la forma de asignar escuelas cambiaría. En ese momento se permitiría que fueran primero las mujeres las que escogieran a donde querían ir a trabajar. Otro profesor manifestó que cuando se le asignó la escuela, el supervisor lo mandó "a capricho” a la escuela más lejana, tal vez "por que le caí mal”.

${ }^{5}$ Regularmente los egresados de normales son gente de clase media baja, que con penurias logran terminar la carrera profesional. Dichos egresados tienen una edad promedio que fluctúa entre los 22 y 24 años.
} 
espera, y que no tiene que ver necesariamente para lo que fueron preparados, es decir, estar frente a grupo y con sus estudiantes (bajo esta aparente tranquilidad y neutralidad que expresa esta última idea) impartiendo clases. ${ }^{6}$

El lugar de asignación de la escuela representa el inicio de un aprendizaje central en la vida del novel profesor. Regularmente son escuelas enclavadas en lo inhóspito de la geografía chiapaneca y son planteles unitarios. Asignada la escuela y la comunidad, el maestro carga su mochila con ilusiones, esperanzas y sobre todo con ánimos por iniciarse; regularmente son muchas horas de viaje en camionetas o en caballo hasta llegar a su destino: la escuela.

La experiencia se empieza a convertir en reveladora. Se inicia por la definición de dónde quedarse a vivir y qué comer, pasando por qué no tendrá las condiciones de su época de estudiante, hasta el conocer la escuela y los estudiantes. La comunidad se ofrece como un cúmulo de experiencias y aprendizajes que el profesor tiene que ir asumiendo. Regularmente no hay quien le diga qué es lo que tiene que hacer, cómo tiene que comportarse. Pero la cautela y los ánimos por granjearse la confianza de la gente hace que el profesor se acomode a las disposiciones y relaciones que se establecen en las comunidades, se sabe que ante la situación compleja no queda más que aguantar, pues está de por medio la seguridad de la plaza laboral. ${ }^{7}$

\footnotetext{
${ }^{6}$ La cuestión a la que se alude es que el aula con estudiantes de comunidades rurales está permeada por una gran cantidad de cuestiones de orden cultural, económicos y sociales, que el profesor principiante tendrá que enfrentar.

7 Uno de los profesores entrevistados señaló lo siguiente: "los primeros días como profesor son de incertidumbre, angustia y tristeza. La soledad es terrible. No está uno preparado para lo que le toca a uno vivir en la primera comunidad. Pero no queda de otra más que aguantar, la necesidad es grande y sabe uno que no se puede perder la plaza”.
}

\section{¿Qué y cómo enseñar?: adaptaciones y aprendizajes docentes}

Ser maestro novel en el contexto rural de Chiapas significa la configuración de un hacer mediado por las circunstancias contextuales, escolares y las de orden personal. Al llegar a la comunidad el maestro tiene en mente que pronto estará en su escuela y frente a sus alumnos. Tiene ánimo de iniciar la tarea para la cual se formó y fue contratado. Se sabe de antemano que el salario llegará tarde (tres o cuatro meses después), al cabo de meses de trámite burocrático, por lo que los primeros meses de ir y venir de la comunidad con la mochila que lo acompaña se complementará con las lágrimas y la soledad. Después de llegado el primer pago, se tendrá un hueco en la mochila para colocar una pequeña despensa de víveres que haga más llevadera la estancia en la comunidad.

Figura 2 - El aula del profesor principiante. Espacio de formación.

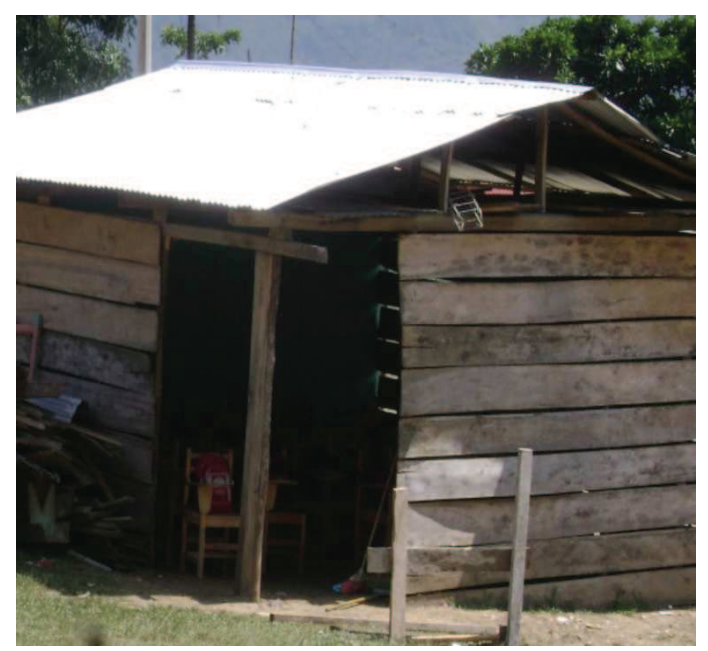

Si es maestro unitario, tendrá que organizar todas las materias y grados. Si es otra modalidad (bidocente o tridocente), 
al menos tendrá compañía, pero no así la posibilidad de descargar la tarea que tiene frente a él. Los maestros noveles se les castigan y "cargan la mano", se les imponen tareas extras por parte de los profesores de más años. El profesor principiante se le da el grado escolar más difícil (según la lógica de los profesores con experiencia), no podrá salir con frecuencia de la comunidad y tendrá que organizar los eventos y festividades cívicas y culturales. De cualquier forma, el profesor principiante, solo o con otros compañeros en su primera escuela, enfrentará un cúmulo de adversidades, tareas que desarrollar que le son ajenas, sin orientación y apoyo para realizarlas.

Ha llegado el momento de enseñar. Pero el ímpetu empieza a chocar con la realidad. Los alumnos de las comunidades presentan problemas serios en cuanto a su desarrollo escolar, pero sobre todo con graves carencias sociales y económicas. Falta de recursos y materiales en las escuelas, y en algunos casos, no se cuenta con una infraestructura que pueda llamar al espacio en donde confluyen alumnos y maestros, como escuela.

En el aula, el maestro se encuentra con que sus alumnos no le entienden y él no entiende a sus alumnos, el ímpetu y la organización (que prepara con esmero y dedicación, en tanto es su carta de presentación) chocan con el hecho de que sus alumnos hablan una lengua indígena.

Llega la etapa de incertidumbre y marcado desconcierto. El qué hacer ante la realidad que se le presenta ronda por la cabeza del maestro, deja pasar las horas y los días hasta considerar opciones que le permitan iniciar su labor, este momento es de intensa soledad, se rememora aquellos tiempos de estudiante en donde tenía un maestro de la escuela Normal que le orientara o le indicara qué hacer, ahora se enfrenta a la realidad de falta de acompañamiento, incluso cuando existen compañeros de trabajo y éstos no sugieren ni apoyan, lo único que hacen es asumir una actitud de fiscalización.

El maestro novel recorre un tramo de su primera incursión profesional entre la improvisación y el diseño de estrategias poco ortodoxas, pero que resultan efectivas. Una profesora entrevistada señalaba que lo común es "tomar al alumno que mejor "medio hable el español” como traductor”. Otro profesor indicaba que no se puede dejar de

considerar que el contenido educativo es poco pertinente, en tanto los antecedentes de referencia de los alumnos no les permite entender lo que se les va a enseñar, por lo que los ajustes son permanentes.

$\mathrm{Y}$ en lo que coinciden todos los profesores es que el grado de marginación y pobreza no les hace "rendir" eficientemente, en tanto la alimentación es precaria, lo que hace que la situación y trabajo docente se complique aun más.

En los primeros días y los primeros meses, el profesor principiante vive con carencias y problemas, pero al conocer la realidad de sus alumnos deja de preocuparse por su situación personal, para inmediatamente pasar a la reflexión de con qué tipo de población está mediando, y qué esa población requiere de su servicio y él no tiene los elementos, referentes y recursos para apoyarlos. Pero de alguna forma se enamorará (MARCELO GARCÍA, 2008, p. 12) y se comprometerá en la relación con sus alumnos, aunque el trabajo de contenidos no sea precisamente el deseado y el que marca el propósito normativo.

Momento de catarsis. Las carencias y problemas que tienen que afrontar los maestros noveles se convierten en acicateadores de reflexión y acción ante la 
complejidad y profundidad que observan de sus alumnos y de la comunidad en su conjunto. $\mathrm{Al}$ momento de llegar a las comunidades y entrar en contacto con los estudiantes y la realidad que los permea, se inicia un proceso de formación y concientización de lo que debe hacer el maestro y también lo que no debe hacer: dejar al garete a los alumnos, ausentarse de la escuela sin justificación, no respetar las costumbres de las comunidades. Proceso de formación que se genera en la vida cotidiana de la presencia en la comunidad, se da a través de la necesidad de resolver las necesidades inmediatas, por salir al paso de las situaciones problemáticas y por llevar a cabo las acciones escolares que sean posibles.

Figura 3 - Alumno de comunidad rural. Motivo para comprometerse en la labor educativa.

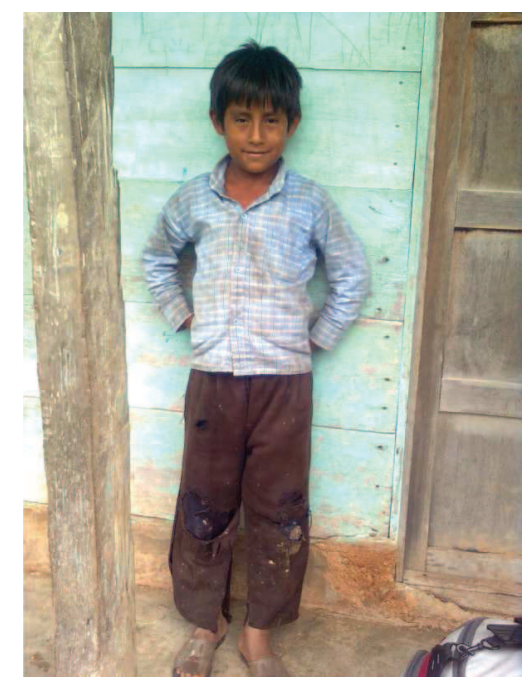

Por lo tanto, es una formación que transcurre en el diario acontecer de los maestros noveles. Formación que da sentido de acción y pensamiento, ubica en su amplia magnitud la labor que deben emprender los maestros de comunidades rurales. En el transcurso del primer año de servicio y hasta el momento en que llega la cadena de cambios y se ubica en una mejor comunidad, el maestro novel pasa de aprender a vivir en la comunidad rural, a entender que su labor va más allá de lo que acontece en las cuatro paredes del aula y que es necesario emprender algunas acciones para intentar que por un momento olviden la complejidad y profundidad de los problemas que enfrentan los niños y jóvenes de dichas comunidades. ${ }^{8}$

\section{Documentar y analizar la configuración del quehacer de los maestros noveles: aprendizaje necesario para plantear rutas de ayuda y mejora.}

Los primeros años en la docencia son especiales en la enseñanza, pero también los son de aprendizaje para el profesor. El primer año, fundamentalmente, comporta elementos que definirán la actuación del profesor en su devenir profesional. Para el caso estudiado, los profesores logran una plaza laboral, incentivada por el anhelo de mejorar la situación personal y familiar de la cual provienen. Mientras llega la plaza, no se repara en las condiciones contextuales que permearán la labor docente. Es hasta el momento de obtener la orden de comisión, que el profesor ve una trasformación de su situación personal y de creciente incertidumbre por enfrentarse a realidades desconocidas y complejas.

\footnotetext{
${ }^{8}$ Para una de las profesoras noveles de educación primaria, "la experiencia del primer año de trabajo fue formativa y reveladora. Pero además adquirió un sentido afectivo de gran envergadura; los niños y adolescentes de estas comunidades no se les puede abandonar a su suerte, requieren tanto apoyo, comprensión y cariño. Como que el estar con ellos, ver su sufrimiento, la pobreza y el olvido, mueven y sacuden la conciencia. Yo me quedé más de un año con ellos, me fue difícil cambiarme, yo sabía que lo podía hacer, pero me esperé un poco de tiempo".
} 
Entre la necesidad laboral y el obtener una plaza, como sea y en las condiciones que sean, se acepta iniciar un recorrido por veredas y montañas, entre la selva $\mathrm{y}$ las comunidades más apartadas de la vida moderna.

El profesor principiante conoce del trabajo en la escuela, sabe de contenidos y programas, pero pronto se dará cuenta que en las comunidades rurales e indígenas eso no basta, es necesario tener conocimientos, habilidades y aptitudes para poder lidiar con el entramado de problemáticas y necesidades que las permea. Por lo anterior, no queda más que participar en lo posible (lo que le da su intuición), improvisando y aplicando una carga importante de dedicación a lo que se realice, aunque no corresponda necesariamente a propósitos educativos. Pasa largas horas viajando para llegar y salir de la comunidad, pues la comunidad representa un mundo lejano a sus interpretaciones y actuaciones y por lo tanto, le es necesario salir para poder tomar una bocanada de civilización.

En los primeros días y meses, el profesor tiene que adaptarse a las condiciones del contexto, aunque la preocupación está latente porque sus alumnos lleguen a la escuela y aprendan, lo cual queda relegado ante el complejo sistema de procesos culturales y sociales que enfrenta.

En la soledad del cuarto donde habita (que le asignan las autoridades de la comunidad), los profesores meditan la realidad que les toca vivir, pero cada día su pensamiento transita hacia la reflexión de las condiciones y los sujetos mismos, a los cuales debe atender en el aula.

La pobreza y marginación de los alumnos, acompañadas de la precariedad en la alimentación y el vestido, la falta de experiencia de ser niño, en tanto están sometidos desde muy pequeños a situaciones que los hacen madurar muy rápidamente, pero también y fundamentalmente el respeto, aprecio y cariño que profesan padres de familia y alumnos hacia los profesores principiantes, hacen que aquilaten y se comprometan en la presencia temporal de su centro de trabajo.

Las condiciones de vida de alumnos y sus familias penetran en el pensamiento del profesorado. Se genera una entrega comprometida por el trabajo áulico, la relación afectiva con el alumnado se profundiza. Pero el profesor carece de "herramientas" para ir más allá del trabajo de los contenidos y establecer un proyecto de intervención educativa que oriente $\mathrm{y}$ ayude en la mejora de las condiciones socioculturales de la gente de la comunidad donde trabaja.

Aunque se asume comprometido, la tarea del profesor se inscribe en el cumplimiento del horario establecido oficialmente, es decir, de lunes a viernes y por un periodo corto de tiempo, ya que el profesor sabe que toda la angustia terminará cuando concluya el ciclo escolar y se genere la cadena de cambios. Las actividades del profesor principiante que van más allá del aula, se inscriben en jugar un deporte con los niños de la escuela o los adultos de la comunidad o caminar con los alumnos por las veredas para apreciar un rio u observar la exuberante flora y fauna (casi aun intocada) que circunda la comunidad.

Al cabo de un año, el profesor está a punto de vivir nuevas experiencias, que ajustarán la práctica profesional que desarrolla. Ya con otra orden de comisión, la de su nuevo centro de trabajo y que es resultado de su solicitud para acercarse a comunidades urbanas con mayores y mejores servicios, y claro para poder alejarse 
de la comunidad en donde "ensayó" y conoció lo que significa ser maestro, y que es la de un maestro que padece inclemencias y carencias, pero sobre todo en donde no hay sanitario, televisión y relaciones con amigos y compañeros de profesión. Ahora tendrá mejores condiciones de vida, pues su antigüedad (un año de servicio) le permite ir escalando en los privilegios del gremio, entre ellos estar ubicado en "una buena escuela”, una comunidad al alcance de la comunicación y los servicios y en mejores condiciones de hospedaje y alimentación.

La experiencia vivida en el primer año de servicio se aquilata, se menciona como inolvidable. Las carencias y vicisitudes calan hondo, definen el pensamiento y la acción, pero contradictoriamente o razonablemente por la forma en que fueron formados, la pretensión es dejar atrás la presencia y actuación como profesor en la primera comunidad y escuela de trabajo.

En la nueva realidad y contexto de trabajo, el profesor aplica la experiencia obtenida, se siente comprometido con la labor, sabe de lo complejo de la realidad educativa de las comunidades apartadas, se comenta entre los colegas lo que se vivió y padeció, pero ha llegado el momento, a partir del cambio de adscripción, de iniciar un nuevo proceso de aprendizaje y adaptación. En la memoria queda solo el recuerdo del primer año de experiencia, "recuerdos que los emocionan y enorgullecen, pero que no siempre reconocen como pasos constitutivos de su identidad profesional" (ALEN; VALERIA, 2009: 12). La idea de entrega y preocupación por la tarea escolar no se borra del pensamiento, así como la situación social y económica de los estudiantes. Un maestro de educación primaria entrevistado señalaba de que "yo hubiera seguido en esa escuela, por mis estudiantes, porque me necesitaban", $\mathrm{y}$ otro refiere "que las condiciones son difíciles, y no estaba preparado para esto”. En el fondo, la cuestión es ir escalando en las mejoras que el gremio otorga e ir participando de las reglas y prácticas escritas y no escritas que permitan mejorar la condición laboral.

Quiero dejar en claro que el problema no radica en dejar la primera escuela, sino que el aprendizaje que se obtiene de la experiencia solo se ubica en el recuerdo y en que "jamás se vuelva a vivir una situación similar”. Otro de los elementos que padecen y carecen los profesores principiantes refiere a los conocimientos y habilidades que les permitan sistematizar y reflexionar lo vivido, por lo que dicha situación lleva a soslayar la vivencia compleja y articuladora de los procesos culturales, sociales y económicos que definen el trabajo en una comunidad rural apartada. No se constituye en un referente para delinear un trabajo docente que recupere la experiencia contextual e histórica. Es indudable que de esa experiencia se reproducirá un saber docente, que le permitirá desarrollar la labor en el aula con mayores referentes, tomando en cuenta el manejo de contenidos y la realidad de los estudiantes; es un saber docente basado en elementos vivenciales que le permitirán ubicar en su real dimensión lo que es la escuela, el profesor principiante regularmente señala "que aquí se adquiere lo que la Normal no enseña”.

Ahora bien, el alejarse de las comunidades apartadas y ubicarse en pueblos comunicados y con servicios, conlleva asumir una posición de profesor entregado a la labor en la nueva escuela de adscripción, participe de comisiones y tareas. Llegó el momento de atender al grupo asignado y el horario establecido, no hay compromiso por la tarde, ni mucho menos contacto con la comunidad más allá del que se establezca por la atención de los alumnos. El profesor asume las reglas no escritas del comportamiento del 
gremio, entre las que destacan la del respeto al horario (no quedarse en la escuela más allá de lo establecido oficialmente), aceptar el "liderazgo" de los profesores con más años de servicio, asumir el acuerdo de ausentarse de la escuela para realizar el cobro de la quincena u otros trámites.

Estas prácticas (entre otras más) se traducirán al poco tiempo en una forma de intervenir y participar en la tarea escolar. No hacerlo implicará trasgredir el proceso de buen funcionamiento de la escuela y su grupo de profesores. El profesor se concentrará en el trabajo delimitado por las cuatro paredes de su aula, más allá de esos límites son terrenos no propicios para mantener su permanencia y aceptación en el grupo.

Es en este contexto que se hace necesario precisar la importancia de investigar y reflexionar la realidad de los profesores principiantes. La cuestión implica considerar que es una etapa que puede definir la ruta de acción del profesional que se inicia en la docencia.

El problema es complejo y demanda una revisión de los procesos formativos que se gestan en las normales, sobre todo el hecho de dotar al estudiante de los elementos que le permitan desenvolverse en cualquier contexto de trabajo. También demanda el establecer la importancia y el valor de los primeros años de servicio. Definir mecanismos de acompañamiento y apoyo. La idea de formación continua y permanente sería base sustantiva en la construcción de la mejora de los procesos de intervención educativa que llevan a cabo los profesores de educación básica en México.

Es necesario definir con claridad roles y perspectivas metodológicas y de interacción educativa, no se puede soslayar la importancia de considerar que para enseñar, es necesario que el profesional posea un cúmulo de conocimientos, habilidades y cualidades humanas que permitan atender y entender a los sujetos con los cuales realizará su labor, pero además para poderse desenvolver en situaciones complejas dentro y fuera del espacio institucional educativo.

Marcelo García (1999) señala la importancia de que el profesor principiante tenga información de la cultura, costumbres, idioma y otros factores acerca de la comunidad, para comprender, sobrevivir y ayudar al desarrollo de la comunidad

Siguiendo a dicho autor, los profesores principiantes debe ubicárseles y ubicarse en la perspectiva de un aprendizaje permanente, continuo, interactivo, acumulativo, mediante un proceso que requiere de elementos multidimensionales y dinámicos, para lograr una dimensión continua, amplia y reflexiva; proceso que lleva al mejoramiento de la práctica docente para lograr la profesionalización de ésta y de la permanencia con gusto y agrado.

La cuestión implica pasar de una formación del profesor de ensayo y error a una en que esté mediando un programa de inducción auspiciado por el Estado y por los actores involucrados en el proceso educativo de los diversos niveles, donde el profesor se le brinde la facilidad de conocer el contexto al que se enfrentará en su práctica profesional y que le permita la interiorización de normas, valores, conductas, actitudes, habilidades para la puesta en marcha de estrategias de enseñanza que sean funcionales en el aprendizaje de los estudiantes a los que atiende.

\section{Comentario final}

La experiencia del profesor principiante en zonas rurales del estado de Chiapas, se encuentra permeada por 
la complejidad de la situación social, económica y cultural en que se encuentra sumida la región. Dichos profesores tienen poca capacidad de maniobra en virtud de los nulos referentes que tienen, como resultado de la formación normalista por la que transitan y que genera una permanente improvisación para sacar adelante el trabajo y que, al final de cuentas, van definiendo acciones y procesos de intervención que les permiten incidir en el aula. La comunidad, el trabajo en ella y la interrelación que se pueda establecer se dejan en un segundo término, en virtud de que no se tiene referentes para intervenir y mucho menos para orientar el desarrollo comunitario.

Es indudable que la experiencia del primer año de ejercicio profesional es formativa, define conocimientos $y$ habilidades que habrán de marcar lo subsecuente de la labor del profesor. Pero las posibilidades de que ese aprendizaje se constituya en eje rector de una formación permanente se ve reducida, en tanto el profesor principiante no tiene los elementos y conocimientos para sistematizarlo, es un aprendizaje por la relación directa, que se asienta en el recuerdo y la memoria, pero que no se convierte en material y pre-texto para la discusión y la reflexión del quehacer docente.

La experiencia del primer año, lo que provoca, por la complejidad y dificultad que representa, es el deseo de jamás volverla a vivir. $\mathrm{Y}$ es que el profesor principiante al no saber y conocer lo que representa una comunidad rural, el proceso de adaptación e integración se hace difícil, se siente ajeno a dicha realidad, y al final de cuentas la experiencia se piensa como elemento que inaugura la práctica de ser profesor, pero dejando de ser "principiante", al cabo de “sobrevivir" el primer ciclo y teniendo antigüedad en el servicio (al menos un año) ya se tiene derechos y es lo que permite ir escalando en los privilegios, entre ellos ir a una mejor comunidad y escuela.

La cuestión demanda reflexionar qué rutas se pueden tomar para aprovechar la experiencia del profesor principiante, es claro que la educación Normal tendría que hacer algo, abrir espacios de formación que permitan conocer el ambiente y realidad de las comunidades rurales. Como también generar un espacio y participación de acompañamiento de profesores noveles, no dejarlos a lo que puedan hacer, desamparados a su suerte.

El maestro recién iniciado en las tareas docentes, regularmente busca cursos y programas de actualización y de formación continua. Como en la región no hay instituciones que le ofrezcan algo relacionado con su actividad, "tiene que ir a caer" a programas académicos que le sean posible financiar y participar (por que la mayoría son en instituciones particulares), regularmente poco tiene que ver con sus necesidades profesionales y solo cubren cuestiones formales. Por lo tanto, necesario es abrir programas de posgrado que recuperen los requerimientos de formación y actualización que ofrezcan opciones de especialización y profundización en torno a la práctica docente, en sus diversas vertientes. Bajo esta lógica, no solo se estaría atendiendo la mejora de la práctica educativa, sino que también la posibilidad del arraigo de los profesores en sus comunidades de trabajo.

El panorama es complejo, pero las posibilidades de mejora son reales. Necesario es revisar la problemática para ir buscando rutas que permitan generar la discusión y abrir los espacios para plantear alternativas. 


\section{Referencias}

ALEN,B.; VALERIA, S. (Coords.). Iniciarse como docente en escuelas rurales. Buenos Aires: Ministerio de Educación, 2009.

BOLIVAR, A. (Coord.). La investigación biográfica-narrativa en educación: enfoque y metodología. Madrid: La muralla, 2001.

MARCELO GARCÍA, C. Estudio sobre estrategias de inserción profesional en Europa. Revista Iberoamericana en Educación, n. 19, p. 1-44. 1999.

MARCELO GARCÍA, C. M. (Coord.). El profesorado principiante. Barcelona: Octaedro, 2008.

SANDOVAL, E. F. (Coord.). Proyecto de investigación: los primeros años de ejercicio docente en educación básica: reconstrucción de prácticas y experiencias en contextos desfavorecidos. México: SEP/ SEB. CONACyT. 146031, 2011.

TARRÉS, M. L. (Coord.). Observar, escuchar y comprender sobre la tradición cualitativa en la investigación social. México: El Colegio de México, FLACSO, Miguel Angel Porrúa, 2004.

Enviado em: 15. Jul. 2012

Aceito em: 22. Nov. 2012 